\title{
ORIGINAL ARTICLE \\ Focal adhesion kinase signaling is decreased 56 days following spinal cord injury in rat gastrocnemius
}

\author{
ZA Graham ${ }^{1,2}$, W Qin ${ }^{1,2}$, LC Harlow ${ }^{1}$, NH Ross ${ }^{1}$, WA Bauman ${ }^{1,2,3}$, PM Gallagher ${ }^{4}$ and CP Cardozo ${ }^{1,2,3,5}$
}

\begin{abstract}
Study design: Descriptive study.
Objectives: The goal of this study was to determine the effects of spinal cord injury (SCI) on aspects of the focal adhesion kinase (FAK) signaling pathway 56 days post injury in rat gastrocnemius.

Setting: This study was conducted in Bronx, NY, USA.

Methods: Three-month-old male Wistar rats were exposed to either a sham surgery $(n=10)$ or complete T4 spinal cord transection $(n=10)$. Rats were killed 56 days following surgery and the muscle was collected. Following homogenization, proteins of the FAK pathway were analyzed by western immunoblotting or reverse transcription-qPCR. In addition, cellular markers for proteins that target the degradation of FAK were investigated.

Results: SCI resulted in significantly lower levels of total and phosphorylated FAK, cSrc and p70S6k, and a trend for increased FRNK protein expression. SCI did not change levels of the $\alpha 7$ or $\beta 1$ integrin subunits, total or phosphorylated ERK1/2, phosphorylated Akt and TSC2 or total p70S6k. SCI resulted in a greater expression of total Akt. mRNA expression of FAK and the $\alpha 7$ or $\beta 1$ integrins remained unchanged between sham and SCl groups. Caspase-3/7 activity and Trim72 mRNA and protein expression remained unchanged following SCl.

Conclusion: $\mathrm{SCl}$ results in diminished FAK signaling and is independent of ERK1/2 and Akt. SCl has no effect on mRNA levels for genes encoding components of the focal adhesion 56 days after injury.
\end{abstract}

Spinal Cord (2016) 54, 502-509; doi:10.1038/sc.2015.183; published online 20 October 2015

\section{INTRODUCTION}

Skeletal muscle is a very adaptable tissue that accounts for a large proportion of organismal mass and has key roles in metabolic homeostasis. The contractile properties of skeletal muscle create postural stability and allow for fine and gross motor movement. Skeletal muscle responds to different types of physical activity, such as aerobic exercise or resistance training, by increases in mass and capacity for oxidative phosphorylation, and hence, exercise is important for improving and maintaining skeletal muscle health., ${ }^{1,2}$ Conversely, the inability to perform physical activity, either acutely such as with a broken bone, or chronically, such as with spinal cord injury (SCI), initiates changes that result in poor muscle and bone health. ${ }^{3}$

There are multiple pathways through which paralysis and reduced physical activity can activate a common downstream pathway for protein catabolism and an increase in transcription of muscle-restricted E3 ubiquitin ligases such as muscle-specific ring finger protein 1 (MuRF1) and muscle atrophy F-box (MAFBx). ${ }^{4}$ Investigating which pro-survival signaling pathways are altered in diseased or immobilized muscle may reveal approaches that can be employed to create therapies to slow or prevent atrophy. The signaling cascade of the $\alpha 7 \beta 1$ integrin is an interesting target in that, as a component of the skeletal muscle costamere, it is a direct sensor of muscle fiber tension ${ }^{5}$ and can initiate pro-survival signaling. ${ }^{6}$ Integrins have no inherent kinase activity and rely on activating focal adhesion kinase (FAK), a non-receptor tyrosine kinase. It has been well established that the state of activation of FAK responds to both severe loading and unloading ${ }^{7-11}$ as well as traditional exercise. ${ }^{12,13}$ FAK may signal through multiple downstream pathways in its role in cellular homeostasis. FAK interacts with $\mathrm{cSrc}$, a $60-\mathrm{kDa}$ protein that is necessary for full FAK activation, ${ }^{14}$ and with extracellular signalrelated kinase $1 / 2$ (ERK 1/2). ${ }^{15}$ FAK can also interact with components of the phosphatidylinositol-3,4 kinase (PI3K)/Akt pathway that suppresses muscle atrophy gene expression programs and stimulate muscle hypertrophy through activation of mTOR and its downstream target p70S6k. ${ }^{16}$

The role of the $\alpha 7 \beta 1$ integrin pathway has only recently been examined in muscle following SCI. Yarar-Fisher et al. ${ }^{17}$ provided interesting evidence that muscle fibers can respond to neuromuscular electrical stimulation resistance training through the $\alpha 7 \beta 1$ integrin pathway in individuals with motor-complete SCI who were studied at an average of 22 years post SCI. We set out to describe the changes in signaling by the $\alpha 7 \beta 1$ integrin FAK pathway subacutely after SCI, and in a more comprehensive manner, by examining signaling through

${ }^{1}$ James J. Peters Veterans Affairs Medical Center, National Center of Excellence for the Medical Consequences of Spinal Cord Injury, Bronx, NY, USA; ${ }^{2}$ Department of Medicine, Icahn School of Medicine at Mount Sinai, New York, NY, USA; ${ }^{3}$ Department of Rehabilitation Medicine, Icahn School of Medicine at Mount Sinai, New York, NY, USA; ${ }^{4}$ Applied Physiology Laboratory, University of Kansas, Lawrence, KS, USA and ${ }^{5}$ Department of Pharmacology and Systems Therapeutics, Icahn School of Medicine at Mount Sinai, New York, NY, USA

Correspondence: Dr CP Cardozo, James J. Peters VA Medical Center, National Center of Excellence for the Medical Consequences of Spinal Cord Injury, 130 West Kingsbridge Road, Bronx, NY 10468, USA.

E-mail: christopher.cardozo@mssm.edu

Received 18 May 2015; revised 21 August 2015; accepted 3 September 2015; published online 20 October 2015 
this pathway in a rat model of SCI caused by a complete spinal cord transection at the fourth thoracic vertebra. Our data suggest that SCI leads to decreases in FAK protein expression and phosphorylation and this is associated with decreases in markers in $\mathrm{mTOR} / \mathrm{p} 70 \mathrm{~S} 6 \mathrm{k}$ signaling independent of ERK $1 / 2$ and Akt.

\section{MATERIALS AND METHODS}

\section{Animals}

Twenty male Wistar rats were used during this study, each weighing $\sim 250 \mathrm{~g}$. These animals were also used in a larger study. ${ }^{18}$ Animals were housed in a facility that was temperature and humidity controlled and had $12 \mathrm{~h}$ day:night cycle. Animals were given access to water and chow ad libitum. All studies with live animals were reviewed and approved by the Institutional Animal Care and Use Committee at the James J. Peters VA Medical Center. Procedures for spinal cord transection and post-operative care have been described in detail in previous publications. ${ }^{19}$ These methods are briefly described below.

\section{Spinal cord injury}

Twenty rats ( $n=10$ per group) underwent a complete transection at the interspace between T3 and T4 (SCI) or sham injury (Sham) in which the spinous process was removed. Post-operative care included administration of antibiotics (Baytril) and analgesics (carprofen) for 3 days post surgery and then as needed. At day 56, the gastrocnemius was excised after the rats were anesthetized via inhalation of 3-5\% isofluorane. Animals were killed via aortic transection.

\section{Protein quantification}

The gastrocnemius was homogenized by an electric tissue homogenizer with a cocktail of cellular lysing buffer, phosphatase and protease inhibitors. The total protein concentration of lysates was determined with a micro-BCA assay (Pierce Scientific, Rockford, IL, USA).
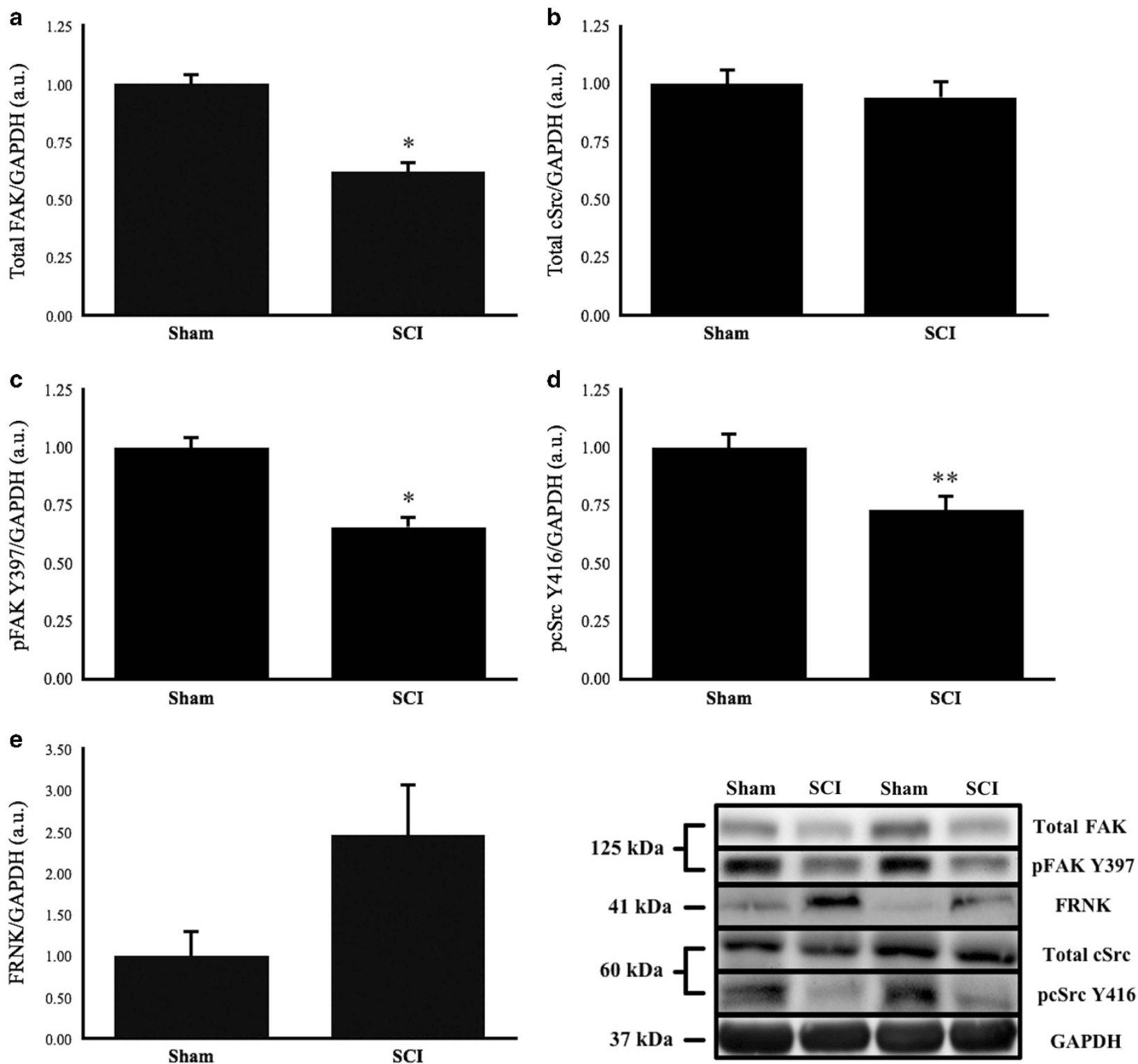

Figure $1 \mathrm{SCl}$ results in decreases in kinase signaling in the rat gastrocnemius. (a) Total FAK expression is decreased 56 days following SCl. (b) Total cSrc expression remains unchanged. (c) Phosphorylated FAK 397 and (d) cSrc Y416 are decreased following SCI. (e) FRNK, a 41-kDa peptide that contains for the FAK C terminus, has a large but non-significant elevation in protein levels post SCl. ${ }^{*}$ Significantly lower expression compared with the Sham group at $P<0.001$. ${ }^{* *}$ Significantly lower expression compared with the Sham group at $P<0.005$. Data are in arbitrary units and mean values \pm s.e.m. 


\section{SDS-PAGE}

Equal amounts of protein $(60 \mu \mathrm{g})$ were mixed 1:1 with Laemlli sample buffer, boiled for $3 \mathrm{~min}$, then loaded into poly-acrylamide gels. Gels were run at a constant $180 \mathrm{~V}$ in $1 \times$ running buffer for $60-90 \mathrm{~min}$.

\section{Western immunoblotting}

Proteins were transferred onto a PVDF membrane by either a semi-dry or wet transfer. Equal protein transfer was confirmed by Ponceau-staining. The membranes were blocked for an hour using 5\% milk and Tris-buffered saline with $0.05 \%$ Tween 20 (TBS-T) then rinsed with TBS-T and incubated overnight at $4{ }^{\circ} \mathrm{C}$ with a primary antibody in a $1 \%$ milk TBS-T solution. All antibodies were purchased from Cell Signaling with the exceptions of Trim72, (Abcam, Cambridge, MA, USA) and C-terminal FAK (used to detect expression of FAK-related non-kinase; FRNK), the $\alpha 7 \beta 1$ subunits and GAPDH (SCBT, Houston, TX, USA). Primary antibody dilutions were 1:500 for phosphorylated and total cSrc and 1:1000 for the rest. Following washing in TBS-T, membranes were incubated with a horseradish peroxidase-conjugated secondary antibody in a $1 \%$ milk TBS-T solution (anti-rabbit or anti-mouse IgG; Cell Signaling, Danvers, MA, USA) at a 1:2000 dilution for an hour. After rinsing membranes in TBS-T, they were incubated with a horseradish peroxidase chemilumenescent reagent (Amersham, Piscataway, NJ, USA). Membranes were then imaged with a digital imaging system (Fluorchem HD2, ProteinSimple; San Jose, CA, USA or Amersham Imager 600, Amersham). Densitometry software (ImageQuant TL v8.1; Amersham) was used to quantify pixel brightness. Each sample was quantified three times and averaged. Samples were normalized to GAPDH.

\section{Real-time qPCR}

Total RNA was extracted from gastrocnemius muscle following homogenization in Tri-Reagent (Sigma-Aldrich, Saint Louis, MO, USA) and quantified by absorbance at $260 \mathrm{~nm}$ with a NanoDrop 1000 (ThermoScientific, Somerset, NJ, USA). All samples had RNA integrity numbers over eight after analysis using an Agilent 2100 Bioanalyzer (Agilent, Santa Clara, CA, USA). The High Capacity cDNA Reverse Transcription Kit (Applied Biosystems, Grand Island, NY, USA) was used to reverse transcribe $1 \mu \mathrm{g}$ of mRNA. Gene expression assays for the $\alpha 7$ (ITGA7) and $\beta 1$ (ITGB1) integrins, FAK (PTK2), Trim72 (TRIM72) and $18 \mathrm{~S}$ ribosomal RNA were purchased from Applied Biosystems. Real-time PCR was performed using Roche FastStart Universal Probe Master Mix and a Viia 7 thermocycler (Applied Biosystems) according to the manufacturer's guidelines.
Relative expression values were calculated using the $2^{-\Delta \Delta C t}$ method. ${ }^{20}$ As the control for these calculations, $18 \mathrm{~S}$ ribosomal RNA was used; $18 \mathrm{~S}$ ribosomal expression was not different between groups.

\section{Caspase-3/7 activity assay}

Caspase-3/7 activity of muscle homogenate was analyzed using a kit according to manufacturer's instructions (Apo-One Homogenous Caspase-3/7; Promega, Madison, WI, USA). Briefly, $40 \mu \mathrm{l}$ of the muscle homogenate supernatant was mixed with the Caspase-3/7 buffer solution in a 1:1 ratio in duplicate in a 96-well white plate and incubated at room temperature for $120 \mathrm{~min}$. The fluorescence intensities of the samples were determined using a BMG Labtech plate reader (Ortenberg, Germany) and analyzed with Mars Data Analysis software (Ortenberg, Germany). Values were normalized to a blank consisting of $40 \mu \mathrm{l}$ of $1 \mathrm{x}$ lysing buffer mixed 1:1 with the Caspase-3/7 buffer solution.

\section{Statistics}

All statistics were carried out using R Software. ${ }^{21}$ One-way multivariate analyses of variance were run for total proteins, phosphorylated proteins and gene expression with significance set at $P<0.050$. Significant one-way multivariate analyses of variance were followed-up with mean tests by an independent samples $t$-test and a Bonferroni correction. The corrected significance for the mean tests was set at $P<0.005$. Caspase-3/7 activity was tested with a two-tailed unpaired samples $t$-test. Data are presented as mean values \pm s.e.m.

We certify that all applicable institutional and governmental regulations concerning the ethical use of animals were followed during the course of this research

\section{RESULTS}

\section{Alterations in FAK signaling and expression}

Following 56 days of hindlimb paralysis caused by SCI, levels of both total and phosphorylated FAK protein were significantly reduced compared with the Sham control group (Figures 1a and c; $P<0.001$ ). Expression of total cSrc protein was unchanged following SCI (Figure $1 b ; P>0.050$ ) but phosphorylation of $c S r c$ at its Y416 activation site was reduced (Figure $1 \mathrm{~d} ; P<0.005$ ). Cleavage of FAK or alternative transcription of the FAK gene can result in expression of a $41-\mathrm{kDa}$ peptide, ${ }^{22}$ known as FRNK, that contains the C-terminal a

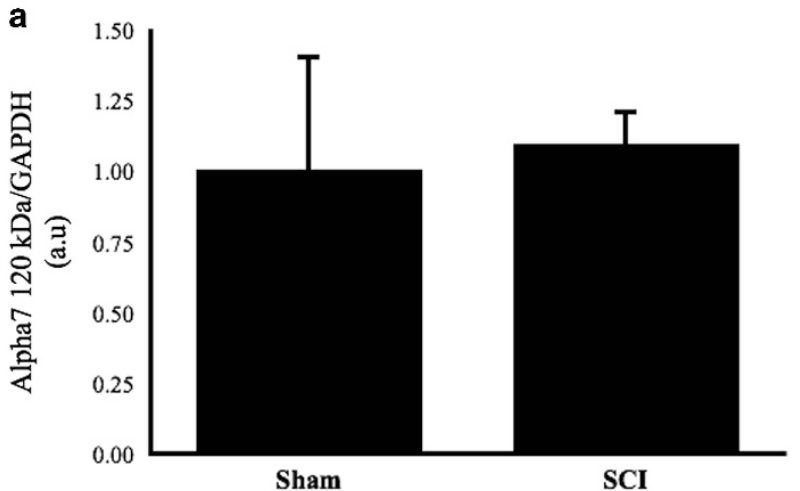

b

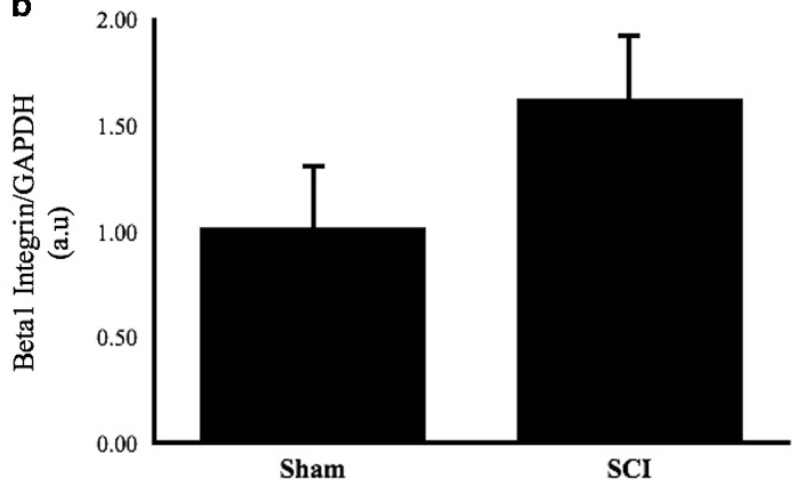

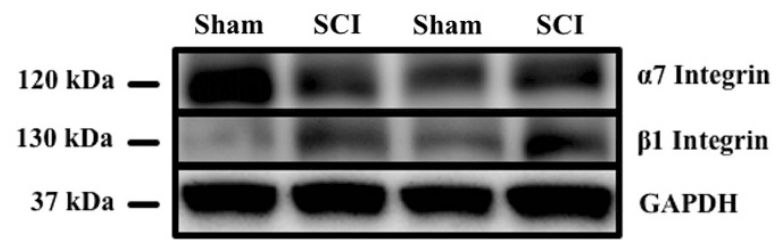

Figure 2 The $\alpha 7 \beta 1$ is the major integrin isoform in mature skeletal muscle. There was no change in protein levels for the (a) $\alpha$ or (b) $\beta$ integrin in rat gastorcnemius muscle 56 days post SCl. Data are in arbitrary units and mean values \pm s.e.m. 
a
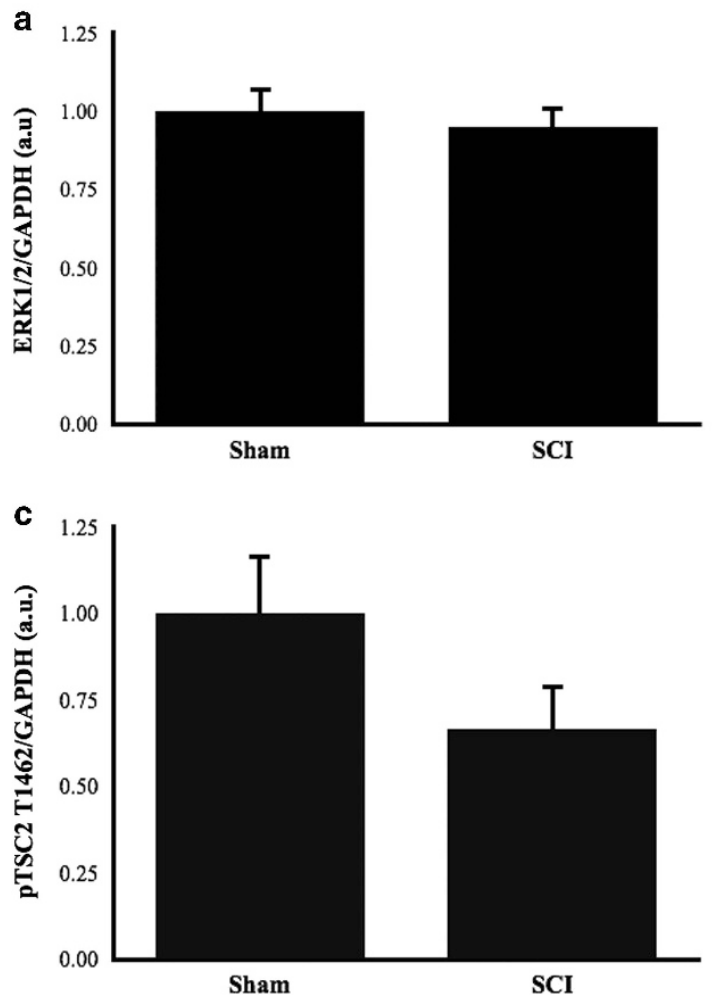

b
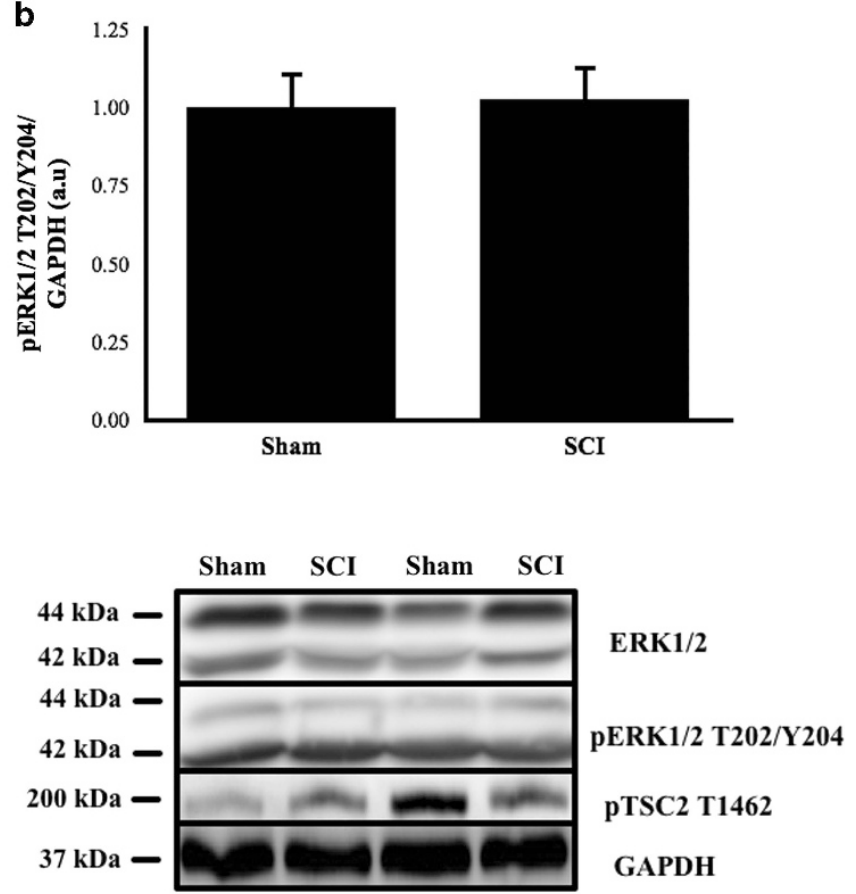

Figure 3 Changes in p70s6k T389 phosphorylation are independent of changes in (a) total ERK1/2 and (b) phosphorylated ERK1/2 T202/Y204. Further, changes in (c) TSC2 T1462 phosphorylation is not different between Sham and SCl groups. Data are in arbitrary units and mean values \pm s.e.m.

portion of FAK. FRNK has been shown to inhibit FAK signaling in muscle. ${ }^{23}$ We saw a large but non-significant increase in FRNK protein expression in the SCI animals compared with the Sham controls (Figure 1e). The role of the $\alpha 7 \beta 1$ integrin as a mechanosensor and structural component of skeletal muscle upstream of FAK led us to investigate whether these two subunits might have altered protein expression 56 days post SCI. However, SCI was not associated with changes in the protein levels of the $\alpha 7 \beta 1$ integrin subunits (Figures $2 \mathrm{a}$ and $\mathrm{b} ; P>0.050)$. The reduction in FAK and cSrc phosphorylation was not associated with changes in total or phosphorylated ERK1/2 T202/Y204 (Figures 3a and b; $P>0.050$ ). TSC2, an inhibitor of mTOR activity of which is inhibited by phosphorylation by ERK1/2 as well as Akt and FAK, showed no change in phosphorylation after SCI (Figure 3c; $P>0.050$ ). Levels of total Akt protein, an important regulator of protein synthesis and glucose metabolism, were elevated after SCI (Figure 4a; $P<0.001$ ) without any change in Akt phosphorylation at $\mathrm{T} 308$ (Figure $4 \mathrm{c} ; \mathrm{P}>0.050$ ), resulting in a numerical change in the phosphorylated:total Akt ratio (data not shown; $P>0.050)$. p70S6k, an important kinase downstream of mTOR, did not have a significant change in total expression after SCI (Figure $4 \mathrm{~b} ; P>0.050$ ) although SCI caused a large reduction in its activating phosphorylation at T389 (Figure 4d; $P<0.001$ ).

\section{Costameric gene expression}

To gain insights into possible mechanisms for the above changes in protein levels, we examined effects of SCI on expression of selected mRNAs for FAK and for the upstream integrin subunits. In addition, we saw no changes in mRNA expression in the SCI group compared with the Sham control group for either integrin subunit (Figures $5 \mathrm{a}$ and $b ; P>0.050)$. FAK mRNA levels were unchanged following 56 days of SCI (Figure $5 c ; P>0.050$ ) suggesting that a post- transcriptional mechanism was involved in reduced levels of FAK after SCI.

\section{Caspase- $3 / 7$ activity and Trim72}

Caspase-mediated FAK cleavage has been implicated in other cell types as being a prominent effector of apoptosis ${ }^{24}$ and has been found to cleave FAK and to participate in cleavage of myofibrillar proteins during muscle atrophy. ${ }^{22}$ To investigate the possibility that caspases are activated in skeletal muscle at 56 days after SCI, activity of caspase 3 and 7 was measured in muscle homogenates using a fluorogenic substrate. The data show that caspase-3/7 activity was not different between the Sham or SCI groups (Figure 6a; $P>0.050$ ). Another alternative explanation for decreased FAK expression is accelerated turnover of FAK by the ubiquitin proteasome pathway. The E3 ubiquitin ligase Trim72 (also known as mitsugumin 53) ubiquitinates FAK and targets it for proteolytic destruction. ${ }^{25}$ Trim72 mRNA and protein levels were, however, not changed at 56 days after SCI (Figure 6b; $P>0.050$ ).

\section{DISCUSSION}

The above findings support the conclusion that FAK protein levels and phosphorylation are reduced following SCI. This alteration was associated with a loss in p70S6K phosphorylation without alterations in phosphorylation of ERK1/2 or Akt. We also provide the first evidence that cSrc phosphorylation is altered in skeletal muscle by paralysis.

The lower levels of expression in FAK and p70S6k phosphorylation observed in rats after SCI are consistent with prior reports that FAK expression and phosphorylation are reduced following other forms of unloading in rats and humans $8,10,11,13$ associated with overall decreases in protein synthesis that are attributed to unloading. ${ }^{10,11}$ Our data 

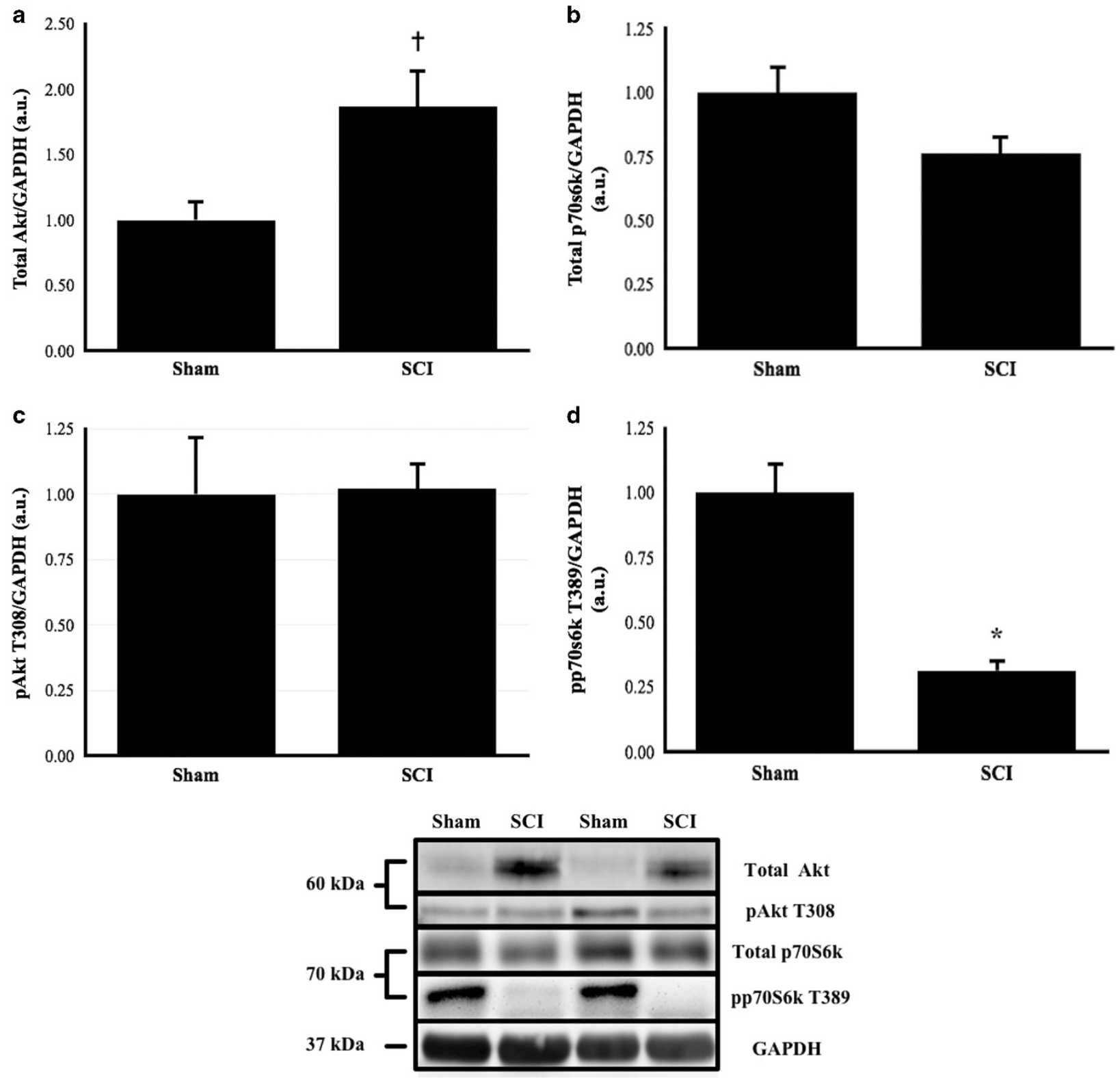

Figure 4 Decreases in active to total Akt ratio may be associated with decreased p70S6k phosphorylation in the gastrocnemius. SCl leads to increases in (a) total Akt, but not (b) total p70s6k. (c) Phosphorylated Akt T308 levels are not affected by SCl. (d) Levels of phosphorylated p70s6k T389 are lower following SCl. 'Significantly greater expression in SCl compared with Sham at $P<0.001$. ${ }^{*}$ Lower expression compared with Sham at $P<0.001$. Data are in arbitrary units and mean values \pm s.e.m.

contrasts with a recent report that in humans, phosphorylated FAK is approximately threefold higher in those with chronic SCI compared with able-bodied controls. ${ }^{17}$ Why FAK would have increased phosphorylation during this state is an intriguing question. Differences between our study and the aforementioned one include species, age and duration of SCI. Their subjects averaged almost 50 years of age and had been injured for, on average, 22 years. The interactions of aging and signaling via the $\alpha 7 \beta 1$ integrin/FAK pathway have not been investigated.

Mechanisms by which absolute levels of FAK protein expression are reduced in muscle after SCI remain unclear. The fact that FAK mRNA levels were unchanged after SCI suggests that either increased protein breakdown or reduced synthesis of FAK was responsible. Although caspases have been shown to target and cleave FAK into inactive fragments, ${ }^{22}$ we found no change in caspase-3/7 activity after SCI, consistent with data from Huey et al. ${ }^{26}$ who saw a return to baseline levels in caspase- 3 activity 28 days post SCI in four different muscle groups. Trim72, a muscle-specific E3 ligase that targets proteins near the membrane following oxidative stress, regulates FAK expression by ubiquitination and proteosomal degradation. ${ }^{25}$ Our data excludes upregulation of this gene as a mechanism underlying the decreases in FAK expression at 56 days post SCI. The numerical though nonsignificant increase in the $41 \mathrm{kDa}$-terminal portion of FAK following SCI suggests that full-length FAK is being cleaved into the inhibitory protein FRNK by an unknown mechanism, although there is a possibility that there is an increase of transcription and translation of FRNK through alternative splicing of FAK mRNA. The decreased levels of FAK protein observed in rat muscle after SCI may be a result 
a

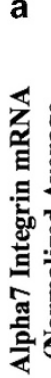

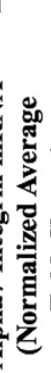

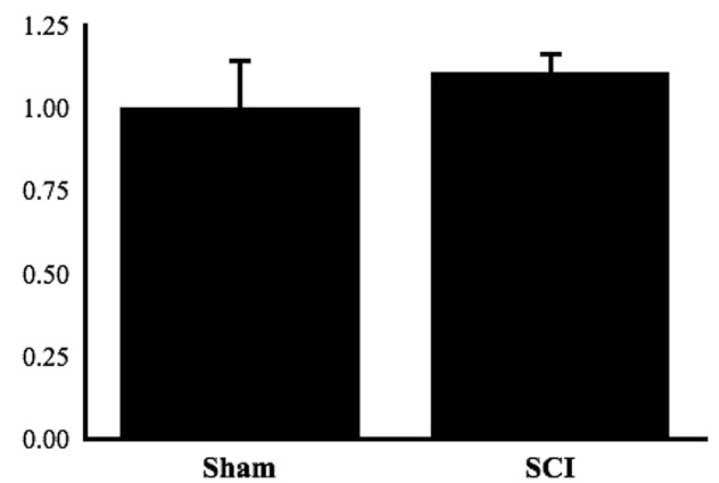

b

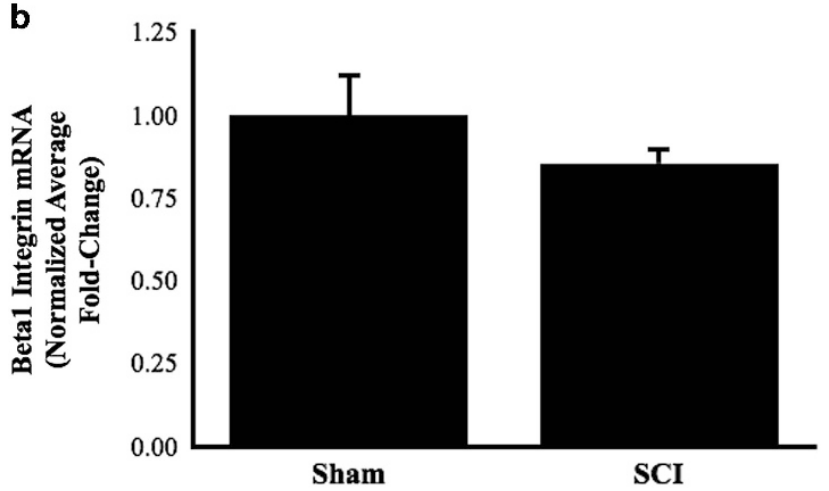

C

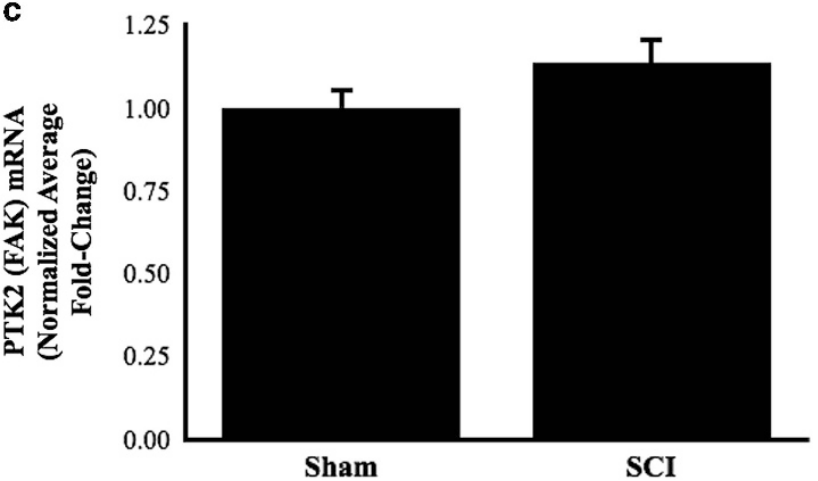

Figure $5 \mathrm{SCl}$ does not change the mRNA levels of genes of the focal adhesion in the rat gastrocnemius. The (a) $\alpha 7$ (ITGA7) or (b) $\beta 1$ (ITGB1) integrin subunits in the rat gastrocnemius do not change 56 days post surgery. Similar results are seen with mRNA expression of (c) FAK (PTK2). Data are presented as normalized fold change and as mean values \pm s.e.m.

of, or contribute to, changes in muscle phenotype. The role of the motor unit in developing and maintaining fiber type has been associated with levels of FAK, as cross-innervating the type 1 soleus with the type 2 dominant extensor digitorum longus results in a decrease in sarcolemmal localization of FAK in rats. ${ }^{27}$ In addition, overexpression of FAK is associated with an increase in markers of an oxidative muscle phenotype. ${ }^{9}$ There is a slow oxidative to fast glycolytic fiber type change following SCI, ${ }^{19,28,29}$ and the loss of type 1 muscle fibers after SCI may be linked to or share common mechanisms with decreases in FAK expression and signaling. This agrees with previous data that shows cytomegalovirus-mediated FAK +FRNK overexpression into the rat soleus is associated with a decrease in type $1 / 2$ muscle fiber hybrids and an increase in type 2 muscle fiber compared with overexpression with FAK alone. ${ }^{23}$
cSrc is a downstream target of FAK, and the reduced phosphorylation of cSrc after SCI is consistent with the corresponding reduction in phospho-FAK levels. The reduction in levels and activation of two key signaling molecules following SCI suggests that integrin-mediated signaling following SCI is greatly impaired. The role of cSrc in skeletal muscle homeostasis has not been well studied. We have recently shown that total and phosphorylated cSrc Y416 were not altered 2 or $48 \mathrm{~h}$ post-eccentric treadmill exercise. ${ }^{30}$ In cardiac myocytes, the FAK/cSrc relationship has been implicated as being an upstream mediator of ERK1/2 activity and hypertrophy following cardiomyocyte overload. ${ }^{31}$ This relationship must be subject to additional regulatory controls, such as changes in levels of phosphatases, because 56 days following SCI reductions in FAK and cSrc phosphorylation were not associated with altered phosphorylation of ERK1/2, even though it is necessary for growth, maintenance and regulation of muscle myotubes $^{32}$ and can be directly related to sarcolemmal tension. ${ }^{33}$ Our findings do agree with evidence that ERK1/2 phosphorylation is unchanged following long-term SCI when compared with able-bodied controls. ${ }^{34}$

It has been long established that FAK activity can be regulated by FRNK by competing for common substrate-binding sites. ${ }^{35,36}$ FAK can activate PI3K, ${ }^{16}$ thereby upregulating, signaling pathways downstream of PI3K to stimulate muscle hypertrophy, namely the PI3K substrate Akt. However, the decrease in FAK phosphorylation observed at 56 days after SCI and increase in FRNK expression were not associated with diminished Akt phosphorylation, perhaps reflecting additional influences on Akt phosphorylation such as receptor tyrosine kinases, growth factor receptors, or altered levels of protein phosphatase activity. Total Akt protein levels were increased, which is consistent with previous reports following $\mathrm{SCI}^{37}$ although the mechanisms for this change are unclear. FAK phosphorylates TSC2 in a region between residues 600 and 1080, and thereby inhibits TSC2 to activate protein synthesis. ${ }^{38}$ In our study, the decreases in FAK phosphorylation did not have an apparent effect on TSC2 phosphorylation. Additional studies to elucidate the actual phosphorylation site and binding mechanisms of TSC2 by FAK would clarify the nature of its TSC2 regulation.

The lack of change in $\alpha 7 \beta 1$ integrin protein levels and mRNA expression suggests that the focal adhesion complex itself is relatively stable 2 months post SCI. Our analysis only looked at the total protein and mRNA expression levels of the $\alpha 7$ and $\beta 1$ integrin subunits. Our findings are, however, consistent with other reports in the literature. Unloading by hindlimb suspension for 3 days does not seem to alter total $\beta 1$ integrin expression in rats. ${ }^{39}$ In humans, $\beta 1$ protein expression increases 10 days after unloading then returns to baseline after 34 days. ${ }^{13}$ There are several splice variants for the alpha7 and $\beta 1$ integrins, the expression of which has been reported to shift from the mature integrin isoforms ( $\alpha 7 \mathrm{X} 2$ and $\beta 1 \mathrm{D}$ integrin, respectively) to the undifferentiated isoforms ( $\alpha 7 \mathrm{X} 1$ and $\beta 1 \mathrm{~A}$ integrin, respectively) during tissue repair after skeletal muscle injury. ${ }^{40}$ How paralysis-induced unloading alters the expression of these isoforms is currently unknown.

Chronic SCI has been associated with reduced activation of mTOR in humans. ${ }^{41}$ Our data suggests that decreased total and phosphorylated expression of FAK may have key roles in this adverse change in mTOR activation. Moreover, our findings suggest that decreased FAK and mTOR activation subacutely after SCI are independent of PI3K/Akt and ERK1/2 signaling. Thus, loading the cytoskeleton of skeletal muscle as soon as possible following SCI may be a way to prevent muscle atrophy through activation of the integrin/FAK pathway. 

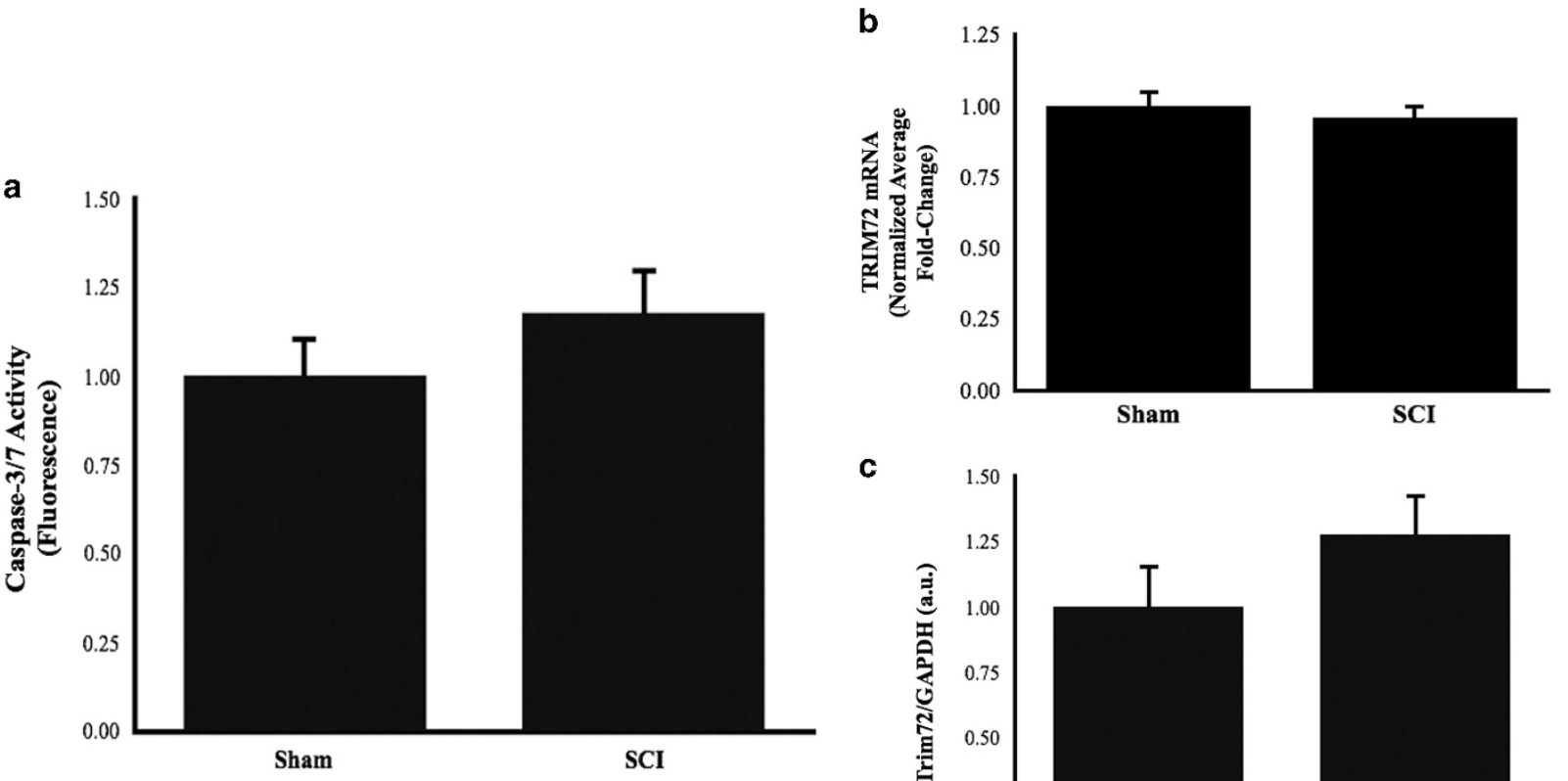

C

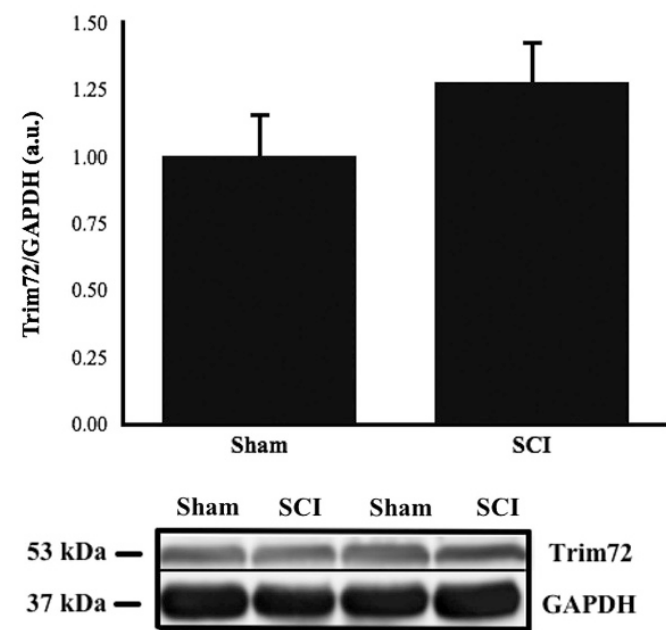

Figure 6 Potential mechanisms for FAK degradation are not altered following 56 days of SCl. (a) Caspase-3/7 activity is not different between Sham or SCl gastrocnemius muscles 56 days post injury. Trim72, an E3 ligase that interacts with membrane-bound proteins and FAK specifically, does not have altered (b) mRNA or (c) protein expression 56 days post SCl. Data are in arbitrary units and mean values \pm s.e.m.

\section{DATA ARCHIVING}

There were no data to deposit.

\section{CONFLICT OF INTEREST}

The authors declare no conflict of interest.

\section{ACKNOWLEDGEMENTS}

This work was supported by the Department of Veterans Affairs Rehabilitation Research and Development Service (B1313R and B0687R) to WQ, (B9212C) to $\mathrm{WAB}$ and $\mathrm{CPC}$, and a University of Kansas General Research Grant to PMG.

1 Baar K, Nader G, Bodine S. Resistance exercise, muscle loading/unloading and the control of muscle mass. Essays Biochem 2006; 42: 61-74.

2 Yan Z, Okutsu M, Akhtar YN, Lira VA. Regulation of exercise-induced fiber type transformation, mitochondrial biogenesis, and angiogenesis in skeletal muscle. J Appl Physiol 2011; 110: 264-274.

3 Qin W, Bauman WA, Cardozo C. Bone and muscle loss after spinal cord injury: organ interactions. Ann NY Acad Sci 2010; 1211: 66-84.

4 Glass DJ. PI3 kinase regulation of skeletal muscle hypertrophy and atrophy. Curr Top Microbiol Immunol 2010; 346: 267-278.

5 Bloch RJ, Gonzalez-Serratos H. Lateral force transmission across costameres in skeletal muscle. Exerc Sport Sci Rev 2003; 31: 73-78.

6 Boppart MD, Burkin D, Kaufman SJ. Activation of AKT signaling promotes cell growth and survival in $\alpha 7 \beta 1$ integrin-mediated alleviation of muscular dystrophy. Biochim Biophys Acta 2011; 1812: 439-6.

7 Fluck M, Carson JA, Gordon SE, Ziemiecki A, Booth FW. Focal adhesion proteins FAK and paxillin increase in hypertrophied skeletal muscle. Am J Physiol 1999; 277: C152-C162.

8 Gordon SE, Fluck M, Booth FW. Selected contribution: skeletal muscle focal adhesion kinase, paxillin, and serum response factor are loading dependent. J Appl Physiol 2001; 90: 1174-1183 (discussion 1165).
9 Durieux AC, D'Antona G, Desplanches D, Freyssenet D, Klossner S, Bottinelli R et al. Focal adhesion kinase is a load-dependent governor of the slow contractile and oxidative muscle phenotype. J Physiol 2009; 587: 3703-3717.

10 de Boer MD, Selby A, Atherton P, Smith K, Seynnes OR, Maganaris CN et al. The temporal responses of protein synthesis, gene expression and cell signalling in human quadriceps muscle and patellar tendon to disuse. J Physiol 2007; 585: 241-251.

11 Glover EI, Phillips SM, Oates BR, Tang JE, Tarnopolsky MA, Selby A et al. Immobilization induces anabolic resistance in human myofibrillar protein synthesis with low and high dose amino acid infusion. J Physiol 2008; 586: 6049-6061.

12 Wilkinson SB, Phillips SM, Atherton PJ, Patel R, Yarasheski KE, Tarnopolsky MA et al. Differential effects of resistance and endurance exercise in the fed state on signalling molecule phosphorylation and protein synthesis in human muscle. J Physiol 2008; 586: 3701-3717.

13 Li R, Narici MV, Erskine RM, Seynnes OR, Rittweger J, Pisot R et al. Costamere remodeling with muscle loading and unloading in healthy young men. J Anat 2013; 223: 525-536.

14 Calalb MB, Polte TR, Hanks SK. Tyrosine phosphorylation of focal adhesion kinase at sites in the catalytic domain regulated kinase activity: a role for the Src family kinases. Mol Cell Biol 1995; 15: 954-963.

15 Chaturvedi LS, Marsh HM, Basson MD. Src and focal adhesion kinase mediate mechanical strain-induced proliferation and ERK1/2 phosphorylation in human $\mathrm{H} 441$ pulmonary epithelial cells. Am J Physiol Cell Physiol 2007; 292: C1701-C1713.

16 Chen HC, Appeddu PA, Isoda H, Guan JL. Phosphorylation of tyrosine 397 in focal adhesion kinase is required for binding phosphatidylinositol 3'-kinase. J Biol Chem 1996; 271: 26329-26334.

17 Yarar-Fisher C, Bickel CS, Kelly NA, Windham ST, McLain AB, Bamman MM. Mechanosensitivity may be enhanced in skeletal muscles of spinal cord-injured versus able-bodied men. Muscle Nerve 2014; 50: 599-601.

18 Qin W, Li X, Peng Y, Harlow LM, Ren Y, Wu Y et al. Sclerostin antibody preserves the morphology and structure of osteocytes and blocks the severe skeletal deterioration after motor-complete spinal cord injury in rats. J Bone Miner Res 2015 (in press).

19 Wu Y, Zhao J, Zhao W, Pan J, Bauman WA, Cardozo CP. Nandrolone normalizes determinants of muscle mass and fiber type after spinal cord injury. J Neurotrauma 2012; 29: 1663-1675.

20 Livak KJ, Schmittgen TD. Analysis of relative gene expression data using real-time quantitative PCR and the 2(-Delta Delta C(T)) Method. Methods 2001; 25: 402-408.

21 R-Core-Team. R: A Language and Environment for Statistical Computing. R Foundation for Statistical Computing, 2014, https://www.r-project.org/. 
22 Gervais FG, Thornberry NA, Ruffolo SC, Nicholson DW, Roy S. Caspases cleave focal adhesion kinase during apoptosis to generate a FRNK-like polypeptide. J Biol Chem 1998; 273: 17102-17108.

23 Klossner S, Li R, Ruoss S, Durieux AC, Fluck M. Quantitative changes in focal adhesion kinase and its inhibitor, FRNK, drive load-dependent expression of costamere components. Am J Physiol Regul Integr Comp Physiol 2013; 305: R647-R657.

24 Mian MF, Kang C, Lee S, Choi JH, Bae SS, Kim SH et al. Cleavage of focal adhesion kinase is an early marker and modulator of oxidative stress-induced apoptosis. Chem Biol Interact 2008; 171: 57-66.

25 Nguyen N, Yi JS, Park H, Lee JS, Ko YG. Mitsugumin 53 (MG53) ligase ubiquitinates focal adhesion kinase during skeletal myogenesis. J Biol Chem 2014; 289: 3209-3216.

26 Huey KA, Roy RR, Zhong H, Lullo C. Time-dependent changes in caspase-3 activity and heat shock protein 25 after spinal cord transection in adult rats. Exp Physiol 2008; 93 : 415-425.

27 Flück M, Ziemiecki A, Billeter R, Müntener M. Fibre-type specific concentration of focal adhesion kinase at the sarcolemma: influence of fibre innervation and regeneration. $J$ Exp Biol 2002; 205: 2337-2348.

28 Roy RR, Zhong H, Monti RJ, Vallance KA, Edgerton VR. Mechanical properties of the electrically silent adult rat soleus muscle. Muscle Nerve 2002; 26: 404-412.

29 Roy RR, Zhong H, Hodgson JA, Grossman EJ, Siengthai B, Talmadge RJ et al. Influences of electromechanical events in defining skeletal muscle properties. Muscle Nerve 2002; 26: 238-251.

30 Graham ZA, Touchberry CD, Gupte AA, Bomhoff GL, Geiger PC, Gallagher PM. Changes in alpha7beta1 integrin signaling after eccentric exercise in heat-shocked rat soleus. Muscle Nerve 2015; 51: 562-568.

31 Torsoni AS, Constancio SS, Nadruz W, Hanks SK, Franchini KG. Focal adhesion kinase is activated and mediates the early hypertrophic response to stretch in cardiac myocytes. Circ Res 2003; 93: 140-147.
32 Shi H, Scheffler JM, Zeng C, Pleitner JM, Hannon KM, Grant AL et al. Mitogen-activated protein kinase signaling is necessary for the maintenance of skeletal muscle mass. Am J Physiol Cell Physiol 2009; 296: C1040-C1048.

33 Martineau LC, Gardiner PF. Insight into skeletal muscle mechanostransduction: MAPK activation is quantitatively related to tension. J Appl Physiol 2001; 91: 693-702.

34 Yarar-Fisher C, Bickel CS, Windham ST, McLain AB, Bamman MM. Skeletal muscle signaling associated with impaired glucose tolerance in spinal cord-injured men and the effects of contractile activity. J Appl Physiol (1985) 2013; 115: 756-764.

35 Nolan K, Lacoste J, Parsons JT. Regulated expression of focal adhesion kinase-related nonkinase, the autonomously expressed $\mathrm{C}$-terminal domain of focal adhesion kinase. Mol Cell Biol 1999; 19: 6120-6129.

36 Richardson A, Parsons T. A mechanism for regulation of the adhesion-associated proteintyrosine kinase pp125FAK. Nature 1996; 380: 538-540.

37 Kim SJ, Roy RR, Kim JA, Zhong H, Haddad F, Baldwin KM et al. Gene expression during inactivity-induced muscle atrophy: effects of brief bouts of a forceful contraction countermeasure. J Appl Physiol (1985) 2008; 105: 1246-1254.

38 Gan B, Yoo Y, Guan JL. Association of focal adhesion kinase with tuberous sclerosis complex 2 in the regulation of s6 kinase activation and cell growth. J Biol Chem 2006; 281: 37321-37329.

39 McClung JM, Thompson RW, Lowe LL, Carson JA. RhoA expression during recovery from skeletal muscle disuse. J Appl Physiol (1985) 2004; 96: 1341-1348.

40 Kääriäinen M, Nissinen L, Kaufman S, Sonnenberg A, Järvinen M, Heino J et al. Expression of alpha7beta1 integrin splicing variants during skeletal muscle regeneration. Am J Pathol 2002; 161: 1023-1031.

41 Dreyer HC, Glynn EL, Lujan HL, Fry CS, DiCarlo SE, Rasmussen BB. Chronic paraplegia-induced muscle atrophy downregulates the mTOR/S6K1 signaling pathway. J Appl Physiol (1985) 2008; 104: 27-33. 